\title{
ESPECIES ACUATICAS DE LOS HUMEDALES DE BOGOTÁ, COLOMBIA CON CAPACIDAD PARA EL TRATAMIENTO DE AGUAS RESIDUALES CON METALES PESADOS: REVIEW
}

\author{
Kenny Pérez de $\operatorname{Arco}^{1} \bowtie$, Johana Castelblanco Lancheros
}

1 Universidad de La Salle, Bogotá.

Colombia $\searrow$ kennyperezdearco @gmail.com

Palabras-clave

Fitorremediación, macrófitas, plantas, acumulación, nativas.

\section{AQUATIC SPECIES OF THE WETLANDS OF BOGOTÁ, COLOMBIA WITH CAPACITY FOR THE TREATMENT OF WASTEWATER WITH HEAVY METALS: REVIEW}

KEY WORDS:

Phytoremediation, macrophytes, plants, accumulation, native.

SUELOS ECUATORIALES 49 (1 y 2): 84-95

ISSN 0562-5351 e-ISSN 2665-6558

\section{RESUMEN}

El presente estudio busca reconocer que especies acuáticas con presencia en los humedales de Bogotá pueden tratar aguas residuales según el tipo de metales pesados y así presentar la información de manera tal que la comunidad pueda generar procesos de diseño más eficientemente. Lo anterior evitar el uso de especies exóticas o que no sean útiles para la retención y/o absorción de ciertos metales pesados. La metodología empleada inicio con una compilación de la información de todas las especies acuáticas con presencia en los humedales de la ciudad, posteriormente se realizó la búsqueda en bases de datos por cada especie sobre la capacidad de bioacumulación de metales pesados y finalmente, se analizó y ordeno la información encontrada.

Como resultado se encontraron 48 especies acuáticas, de las cuales, 24 especies no cuentan con información alguna, 14 requieren una revisión más detallada, 1 especie (utricularia gibba) no puede ser usada en procesos de tratamiento debido a su alta sensibilidad frente a cambios en el ecosistema y especies como holcus lanatus son altamente selectivas con respecto a la resistencia y acumulación de metales pesados, las 24 especies restantes muestran una capacidad variable en la acumulación y se ve afectada por factores como $\mathrm{pH}$ y concentración de metales pesados; se puede concluir que las especies presentes en los humedales de Bogotá no son híper acumuladoras y que se debe incentivar el uso de plantas nativas ya que no actúan como plantas invasoras, evitando así él riesgo de desplazar especies nativas o endémicas.

\section{ABSTRACT}

The present study seeks to recognize that aquatic species with presence in Bogotá wetlands can treat wastewater according to the type of heavy metals and thus present the information in such a way that the community can generate design processes more efficiently. The above avoid the use of exotic species or that are not useful for the retention and / or absorption of certain heavy metals. The methodology used began with a compilation of the information of all aquatic species with presence in the city's wetlands, later the database search was carried out for each species on the capacity of bioaccumulation of heavy metals and finally, it was analyzed and I order the information found.

As result, 48 aquatic species were found, of which 24 species do not have any information, 14 require a more detailed review, 1 species (utricularia gibba) cannot be used in treatment processes due to its high sensitivity to changes in the ecosystem and species such as holcus lanatus are highly selective with respect to resistance and accumulation of heavy metals, the remaining 24 species show a variable capacity in accumulation and is affected by factors such as $\mathrm{pH}$ and concentration of heavy metals; It can be concluded that the species present in the Bogota wetlands are not hyper accumulative and that the use of native plants should be encouraged as they do not act as invasive plants, thus avoiding the risk of displacing native or endemic species..

Rec.: 01.05 .2019

Acep.: 27.06.2019 


\section{INTRODUCCIÓN}

Los humedales son definidos como toda área terrestre que está saturada o inundada de agua de manera estacional o permanente y pueden ser extensiones de marismas, pantanos o turberas cubiertas de agua, sean estas de régimen natural 0 artificial (Ramsar, 2006). Actualmente Bogotá cuenta con 15 humedales registrados, que se caracterizan por ser parte del sistema geográfico del Antiplano Cundiboyacense, el cual pertenece a la cuenca del rio Bogotá y son producto de la desecación paulatina del lago que cubrió el territorio en 1940, con aproximadamente cincuenta mil hectáreas, actualmente solo quedan $727.06 \mathrm{Ha}$ (Moreno, 2015). Estos prestan servicios ecosistémicos como control de inundaciones, reservorio de biodiversidad y depuración de aguas, por mencionar solo algunos.

Sin embargo, de ellos es muy poco lo que se conoce referente a sus especies y la capacidad que tienen estas para emplearse en el tratamiento de aguas residuales. Lo anterior conlleva a que los humedales artificiales (H.A) diseñados y construidos en la ciudad, tengan especies exóticas o que no sean útiles para la retención y/o absorción de ciertos metales pesados, llevando al aumento del tiempo en los pilotajes, al igual que disminución en la eficiencia del tratamiento durante la operación del H.A.

La importancia de la vegetación en los humedales radica en su potencial de adquirir nutrientes y de depurar tóxicos de los afluentes hídricos que recargan los humedales; en Bogotá las principales corrientes hídricas que llegan a los humedales son aguas residuales provenientes de las actividades domésticas 0 industriales que se pueden generar alrededor de los mismos, también las conexiones erradas de la red de alcantarillados de Bogotá que genera esta contaminación a estos ecosistemas.

Por esta razón el objeto del trabajo busca reconocer, que especies acuáticas presentes en los humedales de Bogotá pueden tratar metales pesados presentes en aguas residuales y para llevar a cabo este objeto se compilo la información de todas las especies acuáticas con presencia en los 15 Humedales registrados de la ciudad de Bogotá. Se busco en bases de datos la información reportada por cada especie encontrada con presencia en los humedales de Bogotá, sobre la capacidad de tratar aguas residual y finalmente se analizó y ordeno la información encontrada.

\section{MATERIALES Y METODOS}

El estudio inicio con la Búsqueda y registro de todas las especies de plantas acuáticas exclusivas 0 facultativas (plantas emergentes, flotantes o sumergidas) reportadas en los humedales de la ciudad de Bogotá (Colombia), para esto se emplearon bases de datos a nivel local como la Guía para la identificación de especies de flora presente en los humedales de la sabana de Bogotá, el protocolo de recuperación y rehabilitación ecológica de humedales en centros urbanos 2008, la Guía de plantas acuáticas del jardín de humedales y el Catálogo de plantas invasoras de los humedales de Bogotá.
Seguidamente se verificaron y corrigieron los nombres comunes, científicos, familia y estado de conservación, de las especies acuáticas compiladas, para la verificación y corrección de los nombres comunes, para lo que se empleó la Plataforma de nombre comunes del Jardín Botánico de Bogotá y la Plataforma de Nombres comunes de plantas de Colombia de la Universidad Nacional de Colombia, Para la verificación y corrección de nombres científicos y familia se empleó the Plant list y para verificar el estado de conservación se realizó la consulta en la UICN. 
Una vez compilada esta información se realizó la búsqueda de los artículos publicados para cada especie en la que como parámetro de selección se estableció que a) no presentara otras especies diferente a la evaluada en el estudio y b) no hubiera sido inoculado el medio con bacterias para acelerar o mejorar el proceso. En cada artículo se verifico el uso en la disminución de metales pesados y parámetros fisicoquímicos y el resultado se presenta como la eficiencia de remoción en porcentaje y concentración, para lo cual se empleó la base de datos Science Direct y Scopus, por último, se realizó el análisis de la información.

\section{RESULTADOS Y DISCUSION}

El estudio arrojo que existen 48 especies de plantas acuáticas pertenecientes a 24 familias en los 15 humedales de la ciudad de Bogotá, Colombia; esta información se presenta en la tabla 1, dentro de las cuales las familias más representativas resultaron ser Cyperaceae (12 especies), Poacea (5 especies), onagraceae y araceae (4 especies cada una) y juncácea (3 especies). En su estado de conservación se encontraron 16 especies LC (Preocupación menor), 34 especies NE (No evaluada), 1 especies VU (Vulnerable) y 0 EX (Extinta).

Tabla 1. Especies Acuáticas reportadas en los humedales de Bogotá, Colombia.

\begin{tabular}{|c|c|c|c|}
\hline NOMBRE COMUN & NOMBRE CIENTIFICO & FAMILIA & UICN \\
\hline Pasto de olor & arundo donax & Poaceae & $\mathrm{LC}$ \\
\hline Helecho de Agua & Azolla filiculoides & Salviniaceae & $\mathrm{NE}$ \\
\hline Botoncillo & Bidens laevis & Compositae & $\mathrm{NE}$ \\
\hline Begonia de pantano & callitriche deflexa & Plantaginaceae & $\mathrm{NE}$ \\
\hline Berro de Castilla & carex acutata & Cyperaceae & $\mathrm{NE}$ \\
\hline No encontrado & Carex lasiocarpa & Cyperaceae & $\mathrm{LC}$ \\
\hline No encontrado & carex luridiformis & Cyperaceae & VU A EX LOCAL \\
\hline Cortadera & cotula coronopifolia & Compositae & $\mathrm{NE}$ \\
\hline Cotula & cyperus acuminatus & Cyperaceae & $\mathrm{NE}$ \\
\hline No encontrado & cyperus compactus & Cyperaceae & NE NATIVA \\
\hline Lenteja de Agua & Cyperus papyrus & Cyperaceae & $\mathrm{LC}$ \\
\hline Buchon & Eichhornia crassipes & Pontederiaceae & $\mathrm{NE}$ \\
\hline Cortadera & eleocharis macrostachya & Cyperaceae & $\mathrm{LC}$ \\
\hline Tabaquillo & eleocharis montana & Cyperaceae & $\mathrm{NE}$ \\
\hline Spike rush & eleocharis palustris & Cyperaceae & $\mathrm{NE}$ \\
\hline Junco & eleocharis stenocarpa & Cyperaceae & $\mathrm{NE}$ \\
\hline No encontrado & Equisetum bogotense & equisetaceae & NE NATIVA \\
\hline Cola de caballo & glyceria fluitans & Poaceae & $\mathrm{NE}$ \\
\hline No encontrado & Glyceria septentrionalis & Poaceae & $\mathrm{LC}$ \\
\hline velvet grass & holcus lanatus & Poaceae & $\mathrm{NE}$ \\
\hline Papiro & Hydrocotyle ranunculoides & Araliaceae & LC ENDEMICA \\
\hline Poa & hydrocotyle umbellata & Araliaceae & NE NATIVA \\
\hline Rabo de cachorro & Isolepis inundata & Cyperaceae & $\mathrm{NE}$ \\
\hline Chupana & juncus densiflorus & Juncacea & NE NATIVA \\
\hline Junco & Juncus effusus & Juncacea & $\mathrm{LC}$ \\
\hline Junco & juncus microcephalus & Juncacea & NE NATIVA \\
\hline totorilla & juncus ramboi & salviniaceae & NE NATIVA \\
\hline Lenteja de Agua & Lemna gibba & Araceae & $\mathrm{LC}$ \\
\hline Lenteja de Agua & Lemna minor & Araceae & $\mathrm{LC}$ \\
\hline totorilla & lemna minuta & Araceae & $\mathrm{LC}$ \\
\hline Buchon Cuchara & Limnobium laevigatum & Hydrocharitaceae & $\mathrm{NE}$ \\
\hline
\end{tabular}




\begin{tabular}{llll}
\hline No encontrado & ludwigia arcuata & onagraceae & NE \\
Globito & ludwigia hexapetala & onagraceae & NE NATIVA \\
No encontrado & ludwigia peploides & onagraceae & NE NATIVA \\
Clavito & ludwigia peruviana & onagraceae & NE NATIVA \\
No encontrado & Marsilea ancylopoda & marsiliaceae & NE \\
Trebol de agua & myriophyllum aquaticum & haloragaceae & NE \\
Watercresses & nasturtium officinale & brassicaceae & LC \\
Clavito de agua & persicaria acuminata & Polygonaceae & NE NATIVA \\
berro & Persicaria hydropiperoides & Polygonaceae & LC \\
Barbasco & Polypogon elongatus & Poaceae & NE \\
Utricularia & ranunculus flagelliformis & Ranunculaceae & NE \\
Jacinto de Agua & Schoenoplectus californicus & Cyperaceae & NE NATIVA \\
No encontrado & Spirodela punctata & Araceae & NE \\
Lenteja de Agua & Triglochin scilloides & Juncaginaceae & LC \\
Cumbungi & typha domingensis & thyphaceae & LC \\
Enea & Typha latifolia & Typhaceae & LC NATIVA \\
Southern Cat-tail & utricularia gibba & lentibulariaceae & LC \\
\hline
\end{tabular}

FUENTE: Autores

De las 48 especies de plantas acuáticas con presencia en los humedales de Bogota, se encontró, que no se conoce la capacidad de acumular metales pesados del $50 \%$ de las especies, solo se conoce del $16,6 \%$ de las especies (Tabla 3), el 2,08\% de las especies no puede ser empleada en procesos de remediación y/o acumulación de metales pesados y el $2,08 \%$ de las especies es selectiva con respecto a la resistencia de metales pesados.

Dentro de los metales pesados acumulados de las 48 especies de plantas acuáticas consultadas no se encontró ninguna información para $\mathrm{Hg}$ (II), Tl, Sn y $\mathrm{Ba}$, sin embargo, de la totalidad de las especies especies no se encontró ninguna información asociada a la acumulación de metales pesados para 24 de ellas (Bidens laevis, Carex acutata, C. lasiocarpa, C. luridiformis, Cyperus acuminatus, Eleocharis macrostachya, E. montana, E. palustris, $E$. stenocarpa, Glyceria fluitans, Hydrocotyle ranunculoides, Isolepis inundata, Juncus microcephalus, Lemna minuta, Limnobium laevigatum, ludwigia arcuata, ludwigia hexapetala, ludwigia peploides, ludwigia peruviana, Marsilea ancylopoda, Persicaria acuminata, $P$. hydropiperoides, Polypogon elongatus, Ranunculus scilloides), una especie (holcus lanatus) no tolera el As (Carbonell, 1998) y utricularia gibba, no puede ser empleadas debido a su baja resistencia a la contaminación, por lo que no se debe emplear para procesos de tratamiento, sin embargo, como cualquier cambio en su hábitat conlleva a su desaparición, si puede ser empleada como bioindicador de la calidad de agua.

Adicional a las especies con capacidad de acumulación de metales pesados conocidos, se debe adicionar la capacidad que tiene para mejorar las características físico-químicas (TSS, TDS, DQO, DBO, OD, $\mathrm{SO}_{4}, \mathrm{NO}_{3}, \mathrm{NO}_{2} \mathrm{NH}_{4}$, turbiedad, $\mathrm{pH}$ ), a las cuales se les suman especies como, Callitriche deflexa, Spirodela punctata, Triglochin scilloides, Cyperus papyrus, cotula coronopifolia, cyperus compactus y juncus microcephalus.

El autor (Tarek, 2014) establece una variabilidad en la eficiencia de la especie arundo donax (pasto de olor) al realizar la acumulación de $\mathrm{Cd}$ debido al $\mathrm{pH}$, en los ensayos realizados con $\mathrm{pH}$ de $3.5,5.5$ y 7.5 , obtubo resultados en la eficiencia de remoción de $78 \%, 92 \%$ y $85 \%$, respectivamente, adicionalmente el autor (Shunhui, 2018), afirma que $A$. donax tiene la capacidad de absorción de $\mathrm{Cd}$ y que su acumulación es relativa al aumento de biomasa y crecimiento de la planta, esta especie no es hiperacumuladora y el autor reporta acumulación en tallos, hojas y raíces, sin embargo, la acumulación es significativamente mayor en las raíces.

De acuerdo a (Elfu, 2018) Azolla filiculoides (Helecho de agua), genera rápida disminución de $\mathrm{Cd}$ y Zn y baja remoción en Cd, (Azizur, 2011) menciona la capacidad que esta especie tiene para la remoción de As, $\mathrm{Hg}$ y Cd.

El autor (Azizur, 2011) menciona que la especie Eichhornia crassipes (Buchón) tiene una capacidad para remover $\mathrm{Zn}, \mathrm{Cd}, \mathrm{Cr}, \mathrm{Cu}, \mathrm{Fe}, \mathrm{Ni}, \mathrm{Hg}, \mathrm{Pb}$ y $\mathrm{Zn}$, (Mishra, 2009), adiciona la capacidad que tiene de acumulación de $\mathrm{Cr}$ y $\mathrm{Zn}$ donde se presencia una relación entre eficiencia de remoción y concentración directa, ya que a menor concentración del metal pesado mayor remoción. El autor (Maine, 2009), confirma la eficiencia en la remoción de $\mathrm{Cr}$, al igual 
que muestra una capacidad de remoción de $\mathrm{Ca}$ y $\mathrm{Ni}$, sin embargo, no resulta esta especie ser eficiente en la remoción de Ni ya que presenta una baja eficiencia (5\%), (Maine, 2009) además menciona la capacidad de retención de $\mathrm{Fe}$ al igual que lo reportado por el autor (Mahesh, 2008), adicionalmente el autor (Saima, 2019), encontró una capacidad de remoción de $\mathrm{Cd}, \mathrm{Ni}, \mathrm{Pb}$ y en mayor medida con un $99,9 \%$ de eficiencia en la remoción de Hg.

Con respecto a la especie Equisetum bogotense (Cola de caballo) la autora (Oyuela, 2017), establece una capacidad para la retención y disminución en el agua de As, adicionalmente menciona para la especie juncus densiflorus la capacidad de retención de metales pesados como $\mathrm{Cu}, \mathrm{Pb}$ y $\mathrm{Cd}$.

Para la especie juncus ramboi (totorilla), (Oyuela, 2017) menciona su capacidad de retener $\mathrm{Cu}, \mathrm{Pb}, \mathrm{Cd}$, sin embargo, es necesario realizar estudios con la finalidad de conocer su capacidad real. 
TABLA 2. Especies Acuáticas con capacidad de remoción de metales pesados

\begin{tabular}{|c|c|c|c|c|c|c|c|c|c|c|c|c|c|c|}
\hline ESPECIE & Tipo de agua Residual & Lugar & Unidades & As & $\mathrm{Ca}$ & $\mathrm{Cd}$ & $\mathrm{Cu}$ & $\mathrm{Cr}$ & $\begin{array}{c}\mathrm{Cr} \\
\text { (III) }\end{array}$ & $\begin{array}{c}\mathrm{Cr} \\
(\mathrm{VI})\end{array}$ & Co & $\begin{array}{l}\text { Tiempo } \\
\text { reporte }\end{array}$ & $\begin{array}{c}\mathrm{pH} \\
\text { Agua } \\
\end{array}$ & REFERENCIA \\
\hline \multirow{8}{*}{$\begin{array}{l}\text { arundo } \\
\text { donax }\end{array}$} & \multirow{4}{*}{ Agua Residual Domestica } & \multirow{4}{*}{$\begin{array}{l}\text { Wadi Shueib - } \\
\text { Jordan }\end{array}$} & Concentración (mg/L) & - & - & 20 & - & - & - & - & - & \multirow{4}{*}{$5 \mathrm{hr}$} & & \multirow{4}{*}{ (Tarek, 2014) } \\
\hline & & & Eficiencia remoción (\%) & - & - & 78 & - & - & - & - & - & & 3.5 & \\
\hline & & & Eficiencia remoción (\%) & - & - & 92 & - & - & - & - & - & & 5.5 & \\
\hline & & & Eficiencia remoción (\%) & - & - & 85 & - & - & - & - & - & & 7.5 & \\
\hline & \multirow{4}{*}{$\begin{array}{l}\text { Agua residual artificial con } \\
\text { sulfato de } \mathrm{Cd}\end{array}$} & \multirow{4}{*}{$\begin{array}{l}\text { Wanzhou - } \\
\text { China }\end{array}$} & Concentración $(\mathrm{mg} / \mathrm{L})$ & - & - & 20 & - & - & - & - & - & \multirow{2}{*}{10 dias } & \multirow{4}{*}{6.5} & \multirow{4}{*}{ (Shunhui, 2018) } \\
\hline & & & Eficiencia remoción (\%) & - & - & - & - & - & - & - & - & & & \\
\hline & & & Concentración $(\mathrm{mg} / \mathrm{L})$ & - & - & 0.023 & 0.017 & 0.026 & - & - & 0.038 & & & \\
\hline & & & Eficiencia remoción (\%) & - & - & 23 & 88 & 9 & - & - & 38 & / dias & & \\
\hline \multirow{8}{*}{$\begin{array}{c}\text { Azolla } \\
\text { filiculoides }\end{array}$} & \multirow{4}{*}{$\begin{array}{c}\text { Aguas residuales mezcladas } \\
\text { (textiles, destilería y fuentes } \\
\text { domésticas). }\end{array}$} & \multirow{4}{*}{ Etiopia } & Concentración (mg/L) & - & - & 0.019 & 0.015 & 0.024 & - & - & 0.030 & 14 días & \multirow{4}{*}{$\begin{array}{l}6.5- \\
7.5\end{array}$} & \multirow{4}{*}{ (Elfu, 2018) } \\
\hline & & & Eficiencia remoción (\%) & - & - & 36 & 89 & 19 & - & - & 51 & & & \\
\hline & & & Concentración $(\mathrm{mg} / \mathrm{L})$ & - & - & 0.011 & 0.011 & - & - & - & 0.024 & \multirow{2}{*}{21 días } & & \\
\hline & & & Eficiencia remoción (\%) & - & - & 36 & 92 & - & - & - & 62 & & & \\
\hline & & & Concentración (mg/L) & - & - & 0.010 & - & - & - & - & 0.022 & \multirow{4}{*}{28 días } & & \\
\hline & & & Eficiencia remoción (\%) & - & - & 61 & - & - & - & - & 65 & & & \\
\hline & & & Concentración $(\mathrm{mg} / \mathrm{L})$ & - & - & - & - & 1 & - & - & - & & & \\
\hline & & & Eficiencia remoción (\%) & - & - & - & - & 84 & - & - & - & & & \\
\hline \multirow{13}{*}{$\begin{array}{l}\text { Eichhornia } \\
\text { crassipes }\end{array}$} & \multirow{4}{*}{$\begin{array}{l}\text { Agua residual artificial con } \\
\text { Cr y Zn }\end{array}$} & \multirow{4}{*}{ India } & Concentración (mg/L) & - & - & - & - & 5 & - & - & - & \multirow{4}{*}{$\begin{array}{c}11 \\
\text { días }\end{array}$} & \multirow{4}{*}{8.3} & \multirow{4}{*}{ (Kuma, 2009) } \\
\hline & & & Eficiencia remoción (\%) & - & - & - & - & 79 & - & - & - & & & \\
\hline & & & Concentración (mg/L) & - & - & - & - & 10 & - & - & - & & & \\
\hline & & & Eficiencia remoción (\%) & - & - & - & - & 72 & - & - & - & & & \\
\hline & & & & & & & & & & & & & & \\
\hline & & \multirow{4}{*}{ Argentina } & Concentración (mg/L) & - & - & - & - & 20 & - & - & - & & & \\
\hline & \multirow{8}{*}{$\begin{array}{c}\text { Agua residual industrial } \\
\text { proveniente de una fábrica } \\
\text { de herramientas } \\
\text { agua subterránea de áreas } \\
\text { industriales con hierro }(\mathrm{Fe}) \\
\text { Aguas residuales } \\
\text { combinadas industriales }\end{array}$} & & Eficiencia remoción (\%) & - & - & - & - & 63 & - & - & - & & & \\
\hline & & & Concentración (mg/L) & - & $24-651$ & - & - & $\begin{array}{l}0.001- \\
0.164\end{array}$ & - & - & - & 3 & 9.01 & (Maine 2009) \\
\hline & & & Eficiencia remoción (\%) & - & 34 & - & - & $\begin{array}{l}0.104 \\
53\end{array}$ & - & - & - & años & media & (Mrame, 2009) \\
\hline & & Sri Lanka & Concentración (mg/L) & - & - & - & - & - & - & - & - & 15 & 6.25 & (Mahesh. 2008) \\
\hline & & SII Lälika & Eficiencia remoción (\%) & - & - & - & - & - & - & - & - & SEM & 0.25 & (Mvantesn, 2000) \\
\hline & & Hattar. Pakistán & Concentración (mg/L) & - & - & 0.006 & - & - & - & - & - & 30 & 9.06 & (Saima 2019) \\
\hline & & Hattar, rakistan & Eficiencia remoción (\%) & - & - & 97.5 & - & - & - & - & - & días & 9.00 & (Sarma, 2019), \\
\hline & & & Concentración (mg/L) & 195.9 & - & - & - & - & - & - & - & & & \\
\hline Juncus & & & Eficiencia remoción (\%) & 60 & - & - & - & - & - & - & - & 491 & & \\
\hline effusus & Agua residual sintética & Italia & Concentración $(\mathrm{mg} / \mathrm{L})$ & 146.1 & - & - & - & - & - & - & - & días & 7 & (Salvato, 2010) \\
\hline & & & Eficiencia remoción (\%) & 44 & - & - & - & - & - & - & - & & & \\
\hline
\end{tabular}




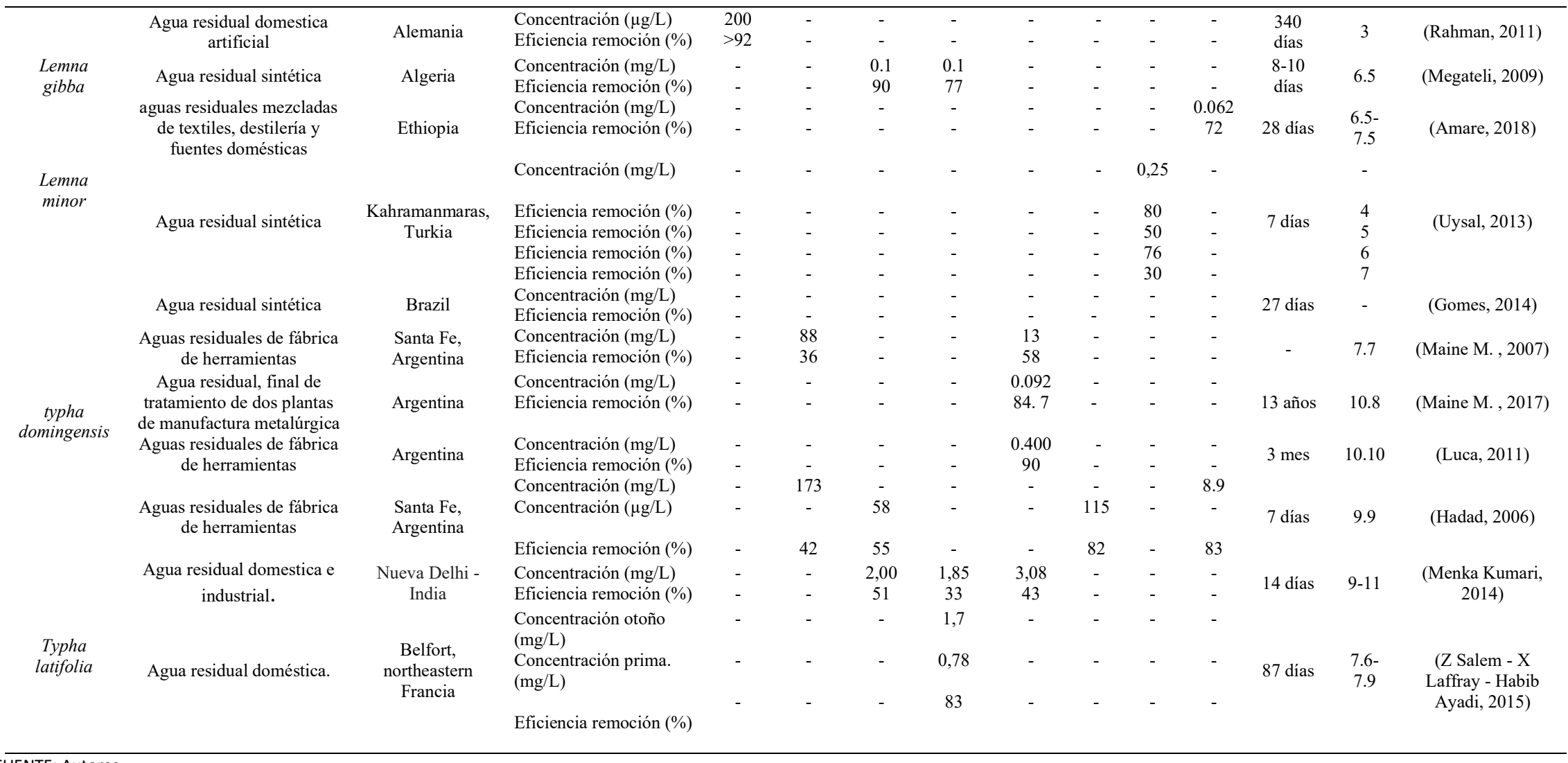


TABLA 3. Especies Acuáticas con capacidad de remoción de metales pesados (CONTINUACION).

\begin{tabular}{|c|c|c|c|c|c|c|c|c|c|c|c|c|c|c|}
\hline ESPECIE & Tipo de agua Residual & Lugar & Unidades & $\mathrm{Fe}$ & $\mathrm{Mg}$ & $\mathrm{Mn}$ & $\mathrm{Ni}$ & $\mathrm{Hg}$ & $\mathrm{Pb}$ & $\begin{array}{l}\mathrm{Pb} \\
\text { (II) }\end{array}$ & $\mathrm{Zn}$ & $\begin{array}{l}\text { Tiempo } \\
\text { reporte }\end{array}$ & $\begin{array}{c}\mathrm{pH} \\
\text { Agua }\end{array}$ & REFERENCIA \\
\hline \multirow{8}{*}{$\begin{array}{l}\text { arundo } \\
\text { donax }\end{array}$} & \multirow{4}{*}{ Agua Residual Domestica } & \multirow{4}{*}{$\begin{array}{l}\text { Wadi Shueib - } \\
\text { Jordan }\end{array}$} & Concentración $(\mathrm{mg} / \mathrm{L})$ & - & - & - & - & - & - & - & - & \multirow{4}{*}{$5 \mathrm{hr}$} & & \multirow{4}{*}{ (Tarek, 2014) } \\
\hline & & & Eficiencia remoción (\%) & - & - & - & - & - & - & - & - & & 3.5 & \\
\hline & & & Eficiencia remoción (\%) & - & - & - & - & - & - & - & - & & 5.5 & \\
\hline & & & Eficiencia remoción (\%) & - & - & - & - & - & - & - & - & & 7.5 & \\
\hline & \multirow{4}{*}{$\begin{array}{l}\text { Agua residual artificial con } \\
\text { sulfato de } \mathrm{Cd}\end{array}$} & \multirow{4}{*}{$\begin{array}{l}\text { Wanzhou - } \\
\text { China }\end{array}$} & Concentración $(\mathrm{mg} / \mathrm{L})$ & - & - & - & - & - & - & - & - & \multirow[b]{2}{*}{10 dias } & \multirow{4}{*}{6.5} & \multirow{4}{*}{ (Shunhui, 2018) } \\
\hline & & & Eficiencia remoción (\%) & - & - & - & - & - & - & - & - & & & \\
\hline & & & Concentración $(\mathrm{mg} / \mathrm{L})$ & 8.684 & - & 0.228 & 0.038 & - & - & - & 0.076 & & & \\
\hline & & & Eficiencia remoción (\%) & 30 & - & 85 & 30 & - & - & - & 87 & 7 días & & \\
\hline \multirow{8}{*}{$\begin{array}{c}\text { Azolla } \\
\text { filiculoides }\end{array}$} & \multirow{4}{*}{$\begin{array}{c}\text { Aguas residuales mezcladas } \\
\text { (textiles, destilería y fuentes } \\
\text { domésticas). }\end{array}$} & \multirow{4}{*}{ Etiopia } & Concentración $(\mathrm{mg} / \mathrm{L})$ & 6.373 & - & 0.214 & - & - & - & - & - & 14tere & \multirow{4}{*}{$\begin{array}{l}6.5- \\
7.5\end{array}$} & \multirow{4}{*}{ (Elfu, 2018) } \\
\hline & & & Eficiencia remoción (\%) & 49 & - & 86 & - & - & - & - & - & 14 dias & & \\
\hline & & & Concentración (mg/L) & 5.386 & - & 0.213 & - & - & - & - & - & \multirow[b]{2}{*}{21 días } & & \\
\hline & & & Eficiencia remoción (\%) & 57 & - & 86 & - & - & - & - & - & & & \\
\hline & & & Concentración (mg/L) & 3.783 & - & 0.200 & - & - & - & - & - & \multirow{4}{*}{28 días } & & \\
\hline & & & Eficiencia remoción (\%) & 70 & - & 87 & - & - & - & - & - & & & \\
\hline & & & Concentración $(\mathrm{mg} / \mathrm{L})$ & - & - & - & - & - & - & - & 1 & & & \\
\hline & & & Eficiencia remoción (\%) & - & - & - & - & - & - & - & 94 & & & \\
\hline \multirow{13}{*}{$\begin{array}{l}\text { Eichhornia } \\
\text { crassipes }\end{array}$} & \multirow{4}{*}{$\begin{array}{l}\text { Agua residual artificial con } \\
\text { Cry Zn }\end{array}$} & \multirow{4}{*}{ India } & Concentración (mg/L) & - & - & - & - & - & - & - & 5 & \multirow{7}{*}{$\begin{array}{c}11 \\
\text { días }\end{array}$} & \multirow{4}{*}{8.3} & \multirow{4}{*}{ (Kuma, 2009) } \\
\hline & & & Eficiencia remoción (\%) & - & - & - & - & - & - & - & 91 & & & \\
\hline & & & Concentración (mg/L) & - & - & - & - & - & - & ـ & 10 & & & \\
\hline & & & Eficiencia remoción (\%) & - & - & - & - & - & - & - & 95 & & & \\
\hline & \multirow{10}{*}{$\begin{array}{c}\text { Agua residual industrial } \\
\text { proveniente de una fábrica } \\
\text { de herramientas } \\
\text { agua subterránea de áreas } \\
\text { industriales con hierro }(\mathrm{Fe}) \\
\text { Aguas residuales } \\
\text { combinadas industriales }\end{array}$} & \multirow[b]{5}{*}{ Argentina } & & & & & & & & & & & & \\
\hline & & & Concentración (mg/L) & - & - & - & - & - & - & - & 20 & & & \\
\hline & & & Eficiencia remoción (\%) & - & - & - & - & - & - & - & 88 & & & \\
\hline & & & Concentración (mg/L) & $0.05-$ & $0.5-59$ & - & $0.002-$ & - & - & - & - & & & \\
\hline & & & Eficiencia remoción (\%) & $\begin{array}{c}73.9 \\
74\end{array}$ & & - & $\begin{array}{l}0.2 \\
39\end{array}$ & - & - & - & - & años & media & (Maine, 2009) \\
\hline & & & Concentración $(\mathrm{mg} / \mathrm{L})$ & 9.27 & - & - & - & - & - & - & - & 15 & & \\
\hline & & Sr1 Lanka & Eficiencia remoción (\%) & 69 & - & - & - & - & - & - & - & SEM & 6.25 & (Mahesh, 2008) \\
\hline & & Hattar Pakistán & Concentración $(\mathrm{mg} / \mathrm{L})$ & - & - & - & 0.161 & 0.003 & 0.199 & - & - & 30 & 9.06 & (Saima, 2019) \\
\hline & & Hattar, Pakistan & Eficiencia remoción (\%) & - & - & - & 95.1 & 99.9 & 83.4 & - & - & días & 9.06 & \\
\hline & & & Concentración (mg/L) & - & - & - & - & - & - & - & - & & & \\
\hline Juncus & & & Eficiencia remoción (\%) & - & - & - & - & - & - & - & - & 491 & & \\
\hline effusus & Agua residual sintética & Italia & Concentración $(\mathrm{mg} / \mathrm{L})$ & - & - & - & - & - & - & - & - & días & 7 & (Salvato, 2010) \\
\hline & & & Eficiencia remoción (\%) & - & - & - & - & - & - & - & - & & & \\
\hline
\end{tabular}




\begin{tabular}{|c|c|c|c|c|c|c|c|c|c|c|c|c|c|c|}
\hline & $\begin{array}{c}\text { Agua residual domestica } \\
\text { artificial }\end{array}$ & Alemania & $\begin{array}{l}\text { Concentración }(\mu \mathrm{g} / \mathrm{L}) \\
\text { Eficiencia remoción }(\%)\end{array}$ & $\begin{array}{l}- \\
-\end{array}$ & $\begin{array}{l}- \\
-\end{array}$ & $\begin{array}{l}- \\
-\end{array}$ & $\begin{array}{l}- \\
-\end{array}$ & $\begin{array}{l}- \\
-\end{array}$ & $\begin{array}{l}- \\
-\end{array}$ & $\begin{array}{l}- \\
-\end{array}$ & $\begin{array}{l}- \\
-\end{array}$ & $\begin{array}{l}340 \\
\text { días }\end{array}$ & 3 & (Rahman, 2011) \\
\hline \multirow{3}{*}{$\begin{array}{l}\text { Lemna } \\
\text { gibba }\end{array}$} & Agua residual sintética & Algeria & Concentración $(\mathrm{mg} / \mathrm{L})$ & - & - & - & - & - & - & - & $\begin{array}{c}0.1 \\
100\end{array}$ & $\begin{array}{l}8-10 \\
\text { dias }\end{array}$ & 6.5 & (Megateli, 2009) \\
\hline & aguas residuales mezcladas & & Concentración $(\mathrm{mg} / \mathrm{L})$ & 12.4 & - & 1.51 & - & - & - & - & 0.6 & & & \\
\hline & $\begin{array}{l}\text { de textiles, destilería y } \\
\text { fuentes domésticas }\end{array}$ & Ethiopia & Eficiencia remoción (\%) & 80 & - & 89 & - & - & - & - & 91 & 28 días & $\begin{array}{c}6.5- \\
7.5\end{array}$ & (Amare, 2018) \\
\hline \multirow{7}{*}{$\begin{array}{l}\text { Lemna } \\
\text { minor }\end{array}$} & & & Concentración $(\mathrm{mg} / \mathrm{L})$ & - & - & - & - & - & - & - & - & & - & \\
\hline & Agua residual sintética & Kahramanmaras, & Eficiencia remoción (\%) & - & - & - & - & - & - & - & - & 7 días & 4 & (Uysal 2013) \\
\hline & Agua residual sintetica & Turkia & Eficiencia remoción (\%) & - & - & - & - & - & - & - & - & t diras & 5 & (Uysal, 2010) \\
\hline & & & $\begin{array}{l}\text { Eficiencia remoción (\%) } \\
\text { Eficiencia remoción }(\%)\end{array}$ & - & - & - & - & - & - & - & - & & $\begin{array}{l}6 \\
7\end{array}$ & \\
\hline & & & Concentración $(\mathrm{mg} / \mathrm{L})$ & - & - & - & - & 0.501 & - & - & - & & & \\
\hline & Agua residual sintetica & Brazıl & Eficiencia remoción (\%) & - & - & - & - & 99.6 & - & - & - & 27 dias & - & (Gomes, 2014) \\
\hline & $\begin{array}{c}\text { Aguas residuales de fábrica } \\
\text { de herramientas }\end{array}$ & $\begin{array}{l}\text { Santa Fe, } \\
\text { Argentina }\end{array}$ & $\begin{array}{l}\text { Concentración }(\mathrm{mg} / \mathrm{L}) \\
\text { Eficiencia remoción }(\%)\end{array}$ & $\begin{array}{c}0.09 \\
80\end{array}$ & $\begin{array}{l}- \\
-\end{array}$ & $\begin{array}{l}- \\
-\end{array}$ & $\begin{array}{l}47 \\
48\end{array}$ & $\begin{array}{l}- \\
-\end{array}$ & $\begin{array}{l}- \\
-\end{array}$ & $\begin{array}{l}- \\
-\end{array}$ & $\begin{array}{l}- \\
-\end{array}$ & - & 7.7 & (Maine M. , 2007) \\
\hline \multirow{6}{*}{$\begin{array}{c}\text { typha } \\
\text { domingensis }\end{array}$} & $\begin{array}{l}\text { Agua residual, final de } \\
\text { tratamiento de dos plantas } \\
\text { de manufactura metalúrgica }\end{array}$ & Argentina & $\begin{array}{l}\text { Concentración }(\mathrm{mg} / \mathrm{L}) \\
\text { Eficiencia remoción }(\%)\end{array}$ & $\begin{array}{l}0.824 \\
89.4\end{array}$ & $\begin{array}{l}- \\
-\end{array}$ & $\begin{array}{l}- \\
-\end{array}$ & $\begin{array}{c}0.048 \\
69.5\end{array}$ & - & $\begin{array}{l}- \\
-\end{array}$ & $\begin{array}{l}- \\
-\end{array}$ & $\begin{array}{c}0.041 \\
51.2\end{array}$ & 13 años & 10.8 & (Maine M. , 2017) \\
\hline & Aguas residuales de fábrica & Argentina & Concentración $(\mathrm{mg} / \mathrm{L})$ & 7.49 & - & - & 0.028 & - & - & - & 0.09 & 3 mes & 10.10 & (Luca, 2011) \\
\hline & de herramientas & Algentina & $\begin{array}{l}\text { Eficiencia remoción (\%) } \\
\text { Concentración }(\mathrm{mg} / \mathrm{L})\end{array}$ & $\begin{array}{c}98 \\
-\end{array}$ & $\overline{24}$ & $\begin{array}{l}- \\
-\end{array}$ & $\begin{array}{c}59 \\
-\end{array}$ & $\begin{array}{l}- \\
-\end{array}$ & $\begin{array}{l}- \\
-\end{array}$ & $\begin{array}{l}- \\
-\end{array}$ & $\begin{array}{l}57 \\
-\end{array}$ & & & \\
\hline & $\begin{array}{c}\text { Aguas residuales de fábrica } \\
\text { de herramientas }\end{array}$ & $\begin{array}{l}\text { Santa Fe, } \\
\text { Argentina }\end{array}$ & Concentración $(\mu \mathrm{g} / \mathrm{L})$ & - & - & - & 175 & - & - & - & 58 & 7 días & 9.9 & (Hadad, 2006) \\
\hline & & & Eficiencia remoción (\%) & - & 39 & - & 69 & - & - & - & 55 & & & \\
\hline & $\begin{array}{c}\text { Agua residual domestica e } \\
\text { industrial. }\end{array}$ & $\begin{array}{l}\text { Nueva Delhi - } \\
\text { India }\end{array}$ & $\begin{array}{l}\text { Concentración }(\mathrm{mg} / \mathrm{L}) \\
\text { Eficiencia remoción (\%) }\end{array}$ & $\begin{array}{c}2,47 \\
34\end{array}$ & $\begin{array}{l}- \\
-\end{array}$ & - & $\begin{array}{l}1,72 \\
39\end{array}$ & - & $\begin{array}{c}1,40 \\
46\end{array}$ & $\begin{array}{l}- \\
-\end{array}$ & $\begin{array}{l}1,70 \\
28\end{array}$ & 14 días & $9-11$ & $\begin{array}{l}\text { (Menka Kumari, } \\
\text { 2014) }\end{array}$ \\
\hline \multirow{3}{*}{$\begin{array}{c}\text { Typha } \\
\text { latifolia }\end{array}$} & & & $\begin{array}{l}\text { Concentración otoño } \\
(\mathrm{mg} / \mathrm{L})\end{array}$ & 2,81 & - & 2,85 & - & - & - & - & - & \multirow{3}{*}{87 días } & \multirow{3}{*}{$\begin{array}{c}7.6- \\
7.9\end{array}$} & \multirow{3}{*}{$\begin{array}{l}\text { (Z Salem - X } \\
\text { Laffray - Habib } \\
\text { Ayadi, 2015) }\end{array}$} \\
\hline & Agua residual doméstica. & northeastern & $\begin{array}{l}\text { Concentración prima. } \\
(\mathrm{mg} / \mathrm{L})\end{array}$ & 0,77 & - & 2,02 & - & - & - & - & - & & & \\
\hline & & & Eficiencia remoción (\%) & 17 & - & 94 & - & - & - & - & - & & & \\
\hline
\end{tabular}


Con respecto a la especie Juncus effusus (junco bogotano), (Salvato, 2010) menciona su capacidad en la retención de As al igual que el autor (Rahman, 2011), este ultimo menciona que la retención de As es mas eficiente en $\mathrm{pHs}$ comprendidos entre 6.6-7.7, sin embargo, encuentra que un $\mathrm{pH}$ comparativamente bajo (3.9-5.9) en la raíz podría mejorar la captación de la planta (en esta especie tiene la capacidad de realizar la acumulación en hojas y raíces, siendo esta ultima la de mayor retencion), además establece su capacidad en el aumento del $\mathrm{pH}$ del agua.

Para Lemna gibba (Lenteja de Agua) el autor (Megateli, 2009), menciona que su gran capacidad para la retención de $\mathrm{Cu}$ y que este se debe a su acción como micronutriente esencial, sin embargo, en grandes concentraciones resulta toxico para la planta, el Cd inhibe el crecimiento de la especie resultando ser el metal mas toxico para L. gibba seguido del $\mathrm{Cu}$ y por último el Zn. Adicional al Zn el autor (Rahman, 2011), menciona su potencial en la acumulación de U y As, además de ser buena acumuladora de $\mathrm{Pb}, \mathrm{Cd}$, $\mathrm{Ni}, \mathrm{Cr}$ y en menor medida $\mathrm{Fe}$, siendo mayor acumuladora en la raíz por sobre las hojas según (Khan, 2009).

Lemna minor (Lenteja de Agua) tiene la capacidad de acuerdo con el autor (Amare, 2018) de acumular Co, $\mathrm{Fe}, \mathrm{Mn}$ y $\mathrm{Zn}$, adicionalmente $\mathrm{Cr}$ y $\mathrm{Ni}$ al igual que $\mathrm{Cd}$ y $\mathrm{Cu}$ de manera similar a L. gibba, el autor (Rahman, 2011), incluye además As y $\mathrm{Hg}$ como metales acumulados por L. minor, de acuerdo con (Iatrou, 2017) esta especie tiene la capacidad de remover medicamentos como benzotriazoles $(1 \mathrm{H}-$ benzotriazole, BTR; 4-methyl-1H-benzotriazole, 4TTR; 5-methyl-1H-benzotriazole, 5TTR; xylytriazole, XTR and 5-chlorobenzotriazole, CBTR), al igual que lo reportado por (Gatidou, 2017). Esta es una de las especies con presencia en los humedales de Bogotá más resistentes a la contaminación, lo que le permite adicionalmente tener una gran capacidad de bioacumulacion de muchos de los metales pesados, sin embargo, se debe tener mucho cuidado en su uso, ya que tiene un comportamiento invasor muy agresivo superando incluso E. crassipes, además el autor (Uysal, 2013) expresa la capacidad de acumulación de $\mathrm{Cr}$ (VI), con una eficiencia entre $50 \%-80 \%$ en un tiempo de 7 días.

Es necesario aclarar aspectos de la especie ludwigia peploides (Duraznillo de agua) ya que el autor (Vymazal, 2013), menciona la presencia de la especie en norte américa, sin embargo, esta también tiene presencia en el sur de américa. El autor (Kamal,
2004), comprobó para la especie Myriophyllum aquaticum (Trebol de agua) una eficiencia de $42.5 \%$ para $\mathrm{Cu}, 75.6 \%$ para $\mathrm{Fe}, 34.4$ para $\mathrm{Zn}$ y $99.9 \%$ para $\mathrm{Hg}$, resultando esta especie eficiente en la acumulación de este ultimo y el autor (Rahman, 2011), menciona que la especie Nasturtium officinale (Watercresses), tiene la capacidad de retención de $\mathrm{Cu}$, Ni y Zn. De acuerdo con cálculos realizados por (Marchand, 2010) la especie schoenoplectus californicus tiene una capacidad de remoción de $80 \%$ $\mathrm{Zn}, 90 \% \mathrm{Hg}, 64 \% \mathrm{Cr}$ y As $96 \%$. Adicionalmente el autor (Sundberg, 2006) menciona la capacidad de esta especie de retención de $\mathrm{Hg}$, Se y As.

El autor (Gomes, 2014) estudio la capacidad de acumulación de $\mathrm{Hg}$ de la especie typha domingensis (junco totora) teniendo una eficiencia en la retención de $99.6 \%$, (Maine M. , 2007), menciona su capacidad para la retención de $\mathrm{Ca}, \mathrm{Cr}, \mathrm{Fe} \mathrm{y} \mathrm{Ni}$, sin embargo, este mismo autor mostro para el año 2017 capacidades de retención de la especie mucho mayor para los metales antes mencionadas además de $\mathrm{Zn}$, esta información es concordante con el autor (Luca, 2011), por lo que se concluye que esta especie genera una muy buena acumulación de metales como $\mathrm{Fe}$, sin embargo se considera en general que typha (genero) tienes una capacidad de acumulación bastante buena de metales como $\mathrm{Fe}$, ademas de tener la capacidad de acumulación de $\mathrm{Cd}, \mathrm{Cr}$ III, Co, Mg según (Hadad, 2006)

La especie Typha Latifolia que menciona el autor (Menka Kumari- B.D Tripathi, 2014), evidencia los valores de concentración de diferentes metales removidos por la captura de 75 litros de agua residual domestica e industrial que fueron usadas para realizar la evaluación de la remoción del mismo durante 14 días; se observa que para metales como el Cobre $(\mathrm{Cu})$, Cadmio (Cd), Cromo (Cr), Niquel (Ni), Hierro ( $\mathrm{Fe}$ ), Plomo $(\mathrm{Pb})$ y Zinc $(\mathrm{Zn})$ hubo porcentajes de remoción de $33,51,43,39,34,46,28$ respectivamente, lo que indica que esta plata tiene la capacidad de filtrar y tratar aguas residuales diferentes metales pesados, principalmente a través de la acción de sus raíces y tejidos en el tallo, como se menciona en el artículo. También se presentan grandes cantidades de remoción de metales como el hierro en aguas residuales domésticas, ya que en el artículo (Z Salem - X Laffray, 2015)referencia una remoción de $80 \%$ en 87 días debido a la captura de este metal a través de las raíces y luego ser transferido a los rizomas de cada planta. 


\section{CONCLUSIONES}

Al momento de realizar procesos de tratamiento de aguas residuales con metales pesados que involucren plantas acuáticas, se debe contemplar aspectos físicoquímicos del agua, siendo uno de los más relevantes el $\mathrm{pH}$ ya que esta influye directamente en la eficiencia de acumulación de la planta, cada especie tiene un rango de $\mathrm{pH}$ en el que su eficiencia aumenta y debe ser un factor decisivo al momento de realizar pruebas piloto de humedales artificiales, siendo un ejemplo, la especie Arundo donax donde trabajar con $\mathrm{pH}$ de 5.5 o superior genera eficiencias por encima del $80 \%$ en la remoción de $\mathrm{Cd}$.

Los procesos de tratamiento en los que existen interacciones bacterianas, junto con plantas acuáticas son procesos de remediación mucho más efectivos en la remoción de metales pesados que los que

\section{BIBLIOGRAFÍA}

Alan Camacho-Mauricio Andrade. (2010). Depuracion de aguas residulaes por medio de humedales artificiales. Bolivia : Centro andino para la gestion y uso del agua .

Amare, E. (2018). Wastewater treatment by Lemna minor and Azolla filiculoides in tropical semi-arid regions of Ethiopia. Ecological Engineering, 464-473.

Azizur, R. M. (2011). Aquatic arsenic: Phytoremediation using floating macrophytes. Chemosphere, 633-646.

Carbonell, A. (1998). Arsenic in wetland vegetation: Availability, phytotoxicity, uptake and effects on plant growth and nutrition. Science of The Total Environment, 189-199.

Convencion sobre los humedales Ramsar. (2015). Estado de los humedales del mundo y de los servicios que prestan a las personas: una recopilacion de a. Nota informativa Ramsar

Diana C. Solano - Javier M. Morales. (2016). Estimacion de la perdida de área en los humedales de Bogotá en las últimas cinco décadas debido a la construcción y sus involucran solamente macrofitas, es necesario evaluar a partir de la información compilada, que tan eficiente resulta el uso de varias especies en un humedal artificial o si por el contrario es mejor el uso de especies por separado.

Las especies de plantas acuáticas que presentan información derivadas de procesos de investigación tienen una distribución mucho mas amplia que las que tienen una presencia a nivel local, por lo que se hace necesario, llevar a cabo procesos de investigación que permitan el desarrollo del conocimiento que conlleven capacidades de remediación empleando especies vegetales locales y a su vez derivando en procesos de conservación y mejora de eficiencias en procesos de tratamientos.

respectivos efectos. Bogotá : Universidad Francisco Jose de Caldas .

Elfu, A. (2018). Wastewater treatment by Lemna minor and Azolla filiculoides in tropical semiarid regions of Ethiopia. Ecological Engineering, 464-473.

Gatidou, G. (2017). Removal mechanisms of benzotriazoles in duckweed Lemna minor wastewater treatment systems. Science of the Total Environment, 12-17.

Gomes, M. V. (2014). Phytoremediation of water contaminated with mercury using Typha domingensis in constructed wetland. Chemosphere, 228-233.

Hadad, H. (2006). Macrophyte growth in a pilot-scale constructed wetland for industrial wastewater treatment. Chemosphere, 17441753.

Iatrou, E. I. (2017). Fate of antimicrobials in duckweed Lemna minor wastewater treatment systems. Journal of Hazardous Materials, 116-126.

Kamal, M. (2004). Phytoaccumulation of heavy metals by aquatic plants. Environment International, 1029-1039. 
Khan, S. (2009). Use of constructed wetland for the removal of heavy metals from industrial wastewater. Journal of Environmental Management, 3451-3457.

Luca, G. D. (2011). Metal retention and distribution in the sediment of a constructed wetland for industrial wastewater treatment. Ecological Engineering, 1267-1275.

Mahesh, W. J. (2008). Contribution of water hyacinth (Eichhornia crassipes (Mart.) Solms) grown under different nutrient conditions to Feremoval mechanisms in constructed wetlands. Journal of Environmental Management, $450-460$.

Maine, M. (2007). Removal efficiency of a constructed wetland for wastewater treatment according to vegetation dominance. Chemosphere, 1105-111.

Maine, M. (2009). Influence of vegetation on the removal of heavy metals and nutrients in a constructed wetland. Journal of Environmental Management, 355-363.

Maine, M. (2017). Long-term performance of two free-water surface wetlands for metallurgical effluent treatment. Ecological Engineering, 372-377.

Marchand, L. (2010). Metal and metalloid removal in constructed wetlands, with emphasis on the importance of plants and standardized measurements: A review. Environmental Pollution, 3447-3461.

Megateli, S. (2009). Toxicity and removal of heavy metals (cadmium, copper, and zinc) by Lemna gibba. Ecotoxicology and Environmental Safety, 1774-1780.

Ministerio de Ambiente y Desarrollo Sostenible. (2018). Bogotá designa los primeros humedales urbanos Ramsar de latinoamerica . Bogotá: Min Ambiente .

Mishra, V. K. (2009). Accumulation of chromium and zinc from aqueous solutions using water hyacinth (Eichhornia crassipes). Journal of Hazardous Materials, 1059-1063.

Oyuela, M. A. (2017). Native herbaceous plant species with potential use in phytoremediation of heavy metals, spotlight on wetlands - A review. Chemosphere, $1230 \mathrm{e} 1247$.

Rahman, K. Z. (2011). Fate and distribution of arsenic in laboratory-scale subsurface horizontal-flow constructed wetlands treating an artificial wastewater. Ecological Engineering, 1214-1224.

Ramsar Convention Secretariat. (8 de 5 de 2014). Funciones ecosistémicas de los humedales. Obtenido de Funciones ecosistémicas de los humedales http://www.ramsar.org/es/acercade/laimportancia-de-los-humedales

Saima, F. (2019). Biological treatment of combined industrial wastewater. Ecological Engineering, 551-558.

Salvato, M. (2010). Effect of different macrophytes in abating nitrogen from a synthetic wastewater. Ecological Engineering, 12221231.

Shunhui, Y. (2018). Physiological response of Arundo donax to cadmium stress by Fourier transform infrared spectroscopy. Spectrochimica Acta Part A: Molecular and Biomolecular Spectroscopy, 88-91.

Sundberg, S. E. (2006). Enrichment of elements in detritus from a constructed wetland and consequent toxicity to Hyalella azteca. Science direct, 264-272.

Tarek, A. (2014). Utilization of a natural ecosystem bio-waste; leaves of Arundo donax reed, as a raw material of low-cost eco-biosorbent for cadmium removal from aqueous phase. Ecological Engineering, 466-473.

Uysal, Y. (2013). Removal of chromium ions from wastewater by duckweed, Lemna minor L. by using a pilot system with continuous flow. Journal of Hazardous Materials, 486492.

Vanessa Moreno-Juan Francisco Garcia. (2015). Descripción general de los humedales de Bogotá . Bogotá : Sociedad Geográfica de Colombia .

Vymazal, J. (2013). Emergent plants used in free water surface constructed wetlands: . Ecological Engineering, 582-592. 